CERN-AB-2008-003 (RF)

CLIC-Note-734

EUROTEV-Report-2008-015

\title{
RF-based electron beam timing measurement with sub-10fs resolution
}

\author{
A. Andersson and J. P. H. Sladen, CERN, Geneva, Switzerland
}

\begin{abstract}
Time of flight measurements of a relativistic electron beam have been performed and have demonstrated a resolution below $10 \mathrm{fs}$. The electronics consisted of a heterodyne receiver incorporating an array of analogue phase detectors in order to reduce noise. The performance of the system makes it suitable for the challenging requirements of intra-pulse train timing measurements in a future linear collider.
\end{abstract}

This paper is a postprint of a paper submitted to and accepted for publication in Electronics Letters and is subject to Institution of Engineering and Technology Copyright. The copy of record is available at IET Digital Library. 


\title{
RF-BASED ELECTRON BEAM TIMING MEASUREMENT WITH SUB-10FS RESOLUTION
}

\author{
A. Andersson and J. P. H. Sladen, CERN, Geneva, Switzerland
}

\begin{abstract}
Time of flight measurements of a relativistic electron beam have been performed and have demonstrated a resolution below $10 \mathrm{fs}$. The electronics consisted of a heterodyne receiver incorporating an array of analogue phase detectors in order to reduce noise. The performance of the system makes it suitable for the challenging requirements of intra-pulse train timing measurements in a future linear collider.
\end{abstract}

\section{INTRODUCTION}

The electronics for a precision beam phase measurement have been developed and tested at CERN's CTF3 [1] accelerator. The motivation for this is the stringent requirements for the measurement and control of beam timing in a future linear collider such as CLIC (Compact Linear Collider) [2] where precision in the order of $10 \mathrm{fs}$ will be required.

A heterodyne approach has been taken, measuring the phase of the $10^{\text {th }}$ harmonic of the $3 \mathrm{GHz}$ bunch frequency in CTF3. The $30 \mathrm{GHz}$ component of the beam was sampled in a pick-up and then mixed down to an IF of $750 \mathrm{MHz}$. A wideband phase measurement of $50 \mathrm{MHz}$ or greater is required in order to provide intra-pulse timing information. This, together with the need for low noise, precludes the use of digital phase detection at present. Instead, an array of analogue multipliers was used with their outputs summed and averaged. Preliminary tests [3] yielded promising results but highlighted several imperfections in the system that have now been addressed.

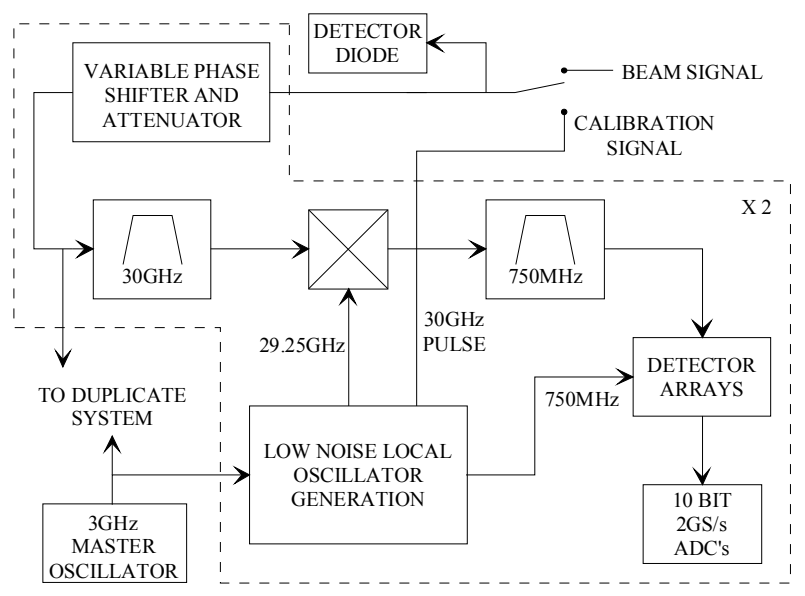

Fig. 1 Test set-up

\section{MEASUREMENT SET-UP}

The basic test set-up for beam measurements (Fig. 1) has already been described elsewhere [3] so only brief details are given here. As the beam jitter far exceeded the expected resolution, two systems were installed and their outputs compared. The beam signal input for both systems was identical, coming from a $30 \mathrm{GHz}$ power extraction structure [4] installed in a CTF3 beam line. Variable attenuators and phase shifters at the inputs were used for adjusting power levels and for setting phases to within the measurement window. The input for the second system was taken after the phase shifter and attenuator of the first, thus permitting both common and independent adjustments. The signal was then mixed down using a low jitter local oscillator derived from CTF3's $3 \mathrm{GHz}$ master clock. The phase detection electronics consisted of a set of power splitters feeding an array of eight AD835 analogue multipliers with summed outputs. Input signal power was not a limitation and this parallelisation and summation permitted a reduction of the multiplier noise through averaging. An array of eight amplitude detectors was also used. After digitization, the amplitude information was used to correct for the amplitude dependence of the phase detector. System bandwidth was limited by a $30 \mathrm{GHz}$ input waveguide filter to a maximum of $\pm 150 \mathrm{MHz}$. Dynamic range for low noise measurements was $10^{\circ}$ (corresponding to $0.93 \mathrm{ps}$ at $30 \mathrm{GHz}$ ), limited by the ADC's.

The improvements to the original set-up included better linearity in the IF amplification and a higher power calibration signal. The latter permitted the two systems to be calibrated offline with a $30 \mathrm{GHz}$ pulse over the required amplitude range. Of interest here is to demonstrate that the calibration can be used to accurately align the responses of the two systems with beam.

\section{RESULTS}

Results were obtained with $90 \mathrm{MeV}, 4 \mathrm{~A}$ beam pulses of length $100 \mathrm{~ns}$ and $333 \mathrm{ps}$ bunch spacing. Beam conditions, adjusted for $30 \mathrm{GHz}$ power production, were not ideal for precision phase measurements. In particular, the phase variation across the pulse was always too large (between $30^{\circ}$ and $100^{\circ}$ ) and so exceeded the dynamic range available for low noise measurements. This precluded low noise acquisition of the complete pulse. Consequently, jitter and pulse response measurements were performed separately. The noise measurements were done over a portion of the $100 \mathrm{~ns}$ pulse fitting into a $10^{\circ}$ 
window. The measurement range was then increased for the pulse response measurements. The additional noise introduced was not important for these measurements as averaged data could be used.

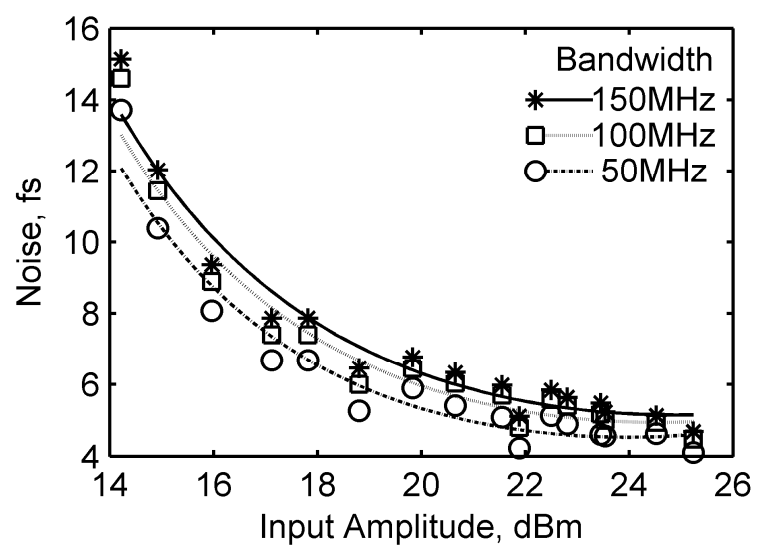

Fig. 2 Beam timing measurement jitter

Jitter measurements are shown in Fig. 2 for an $11 \mathrm{~dB}$ range of input amplitudes. The values correspond to $\sqrt{2}$ of the measured jitter between the two systems and include local oscillator, down-conversion, phase detection and digitization noise as well as post-detection noise introduced by a third order correction for the phase detector output dependence on input amplitude. Slow temperature drift during the measurements $\left(110 \mathrm{fs} /{ }^{\circ} \mathrm{C}\right)$ has been removed. Jitter below $10 \mathrm{fs}$ is obtained over a $9 \mathrm{~dB}$ dynamic range.

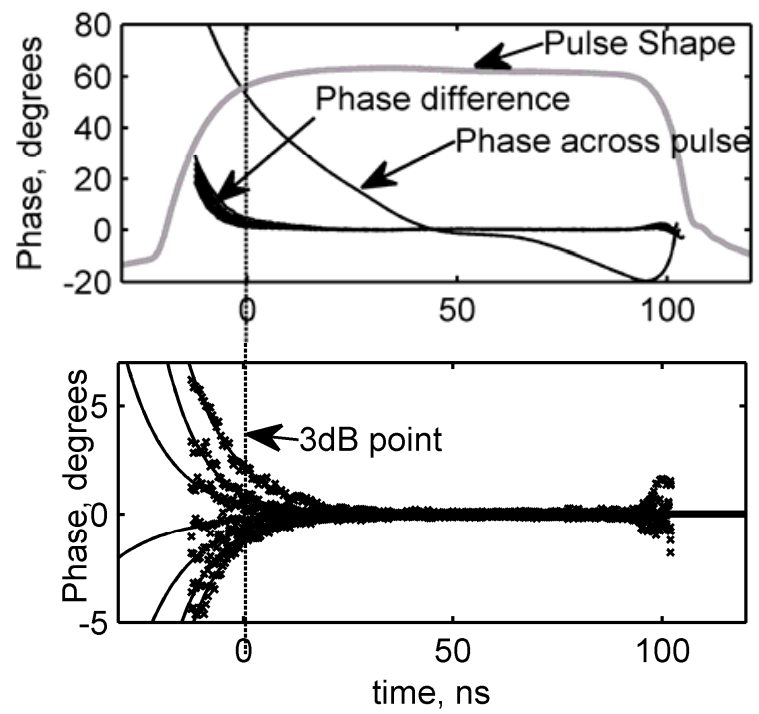

Fig. 3 Response to beam pulse

Averaged data for the complete beam pulse are shown in Fig. 3. As well as pulse shape and phase profile, the top plot shows the measured phase difference between the two systems for seven different amplitude levels over a $5.4 \mathrm{~dB}$ range. They are shown more clearly in the lower plot where the mean phase across the full range has been removed. The offline calibration can be seen to result in very good alignment between the two systems. Its accuracy improves as the amplitude range is reduced. This is shown in Fig. 4 where the error along the pulse (starting at the $-3 \mathrm{~dB}$ point) is plotted for six different amplitude ranges. The error value is that of the worst case curve within the amplitude range with respect to the mean of the range.

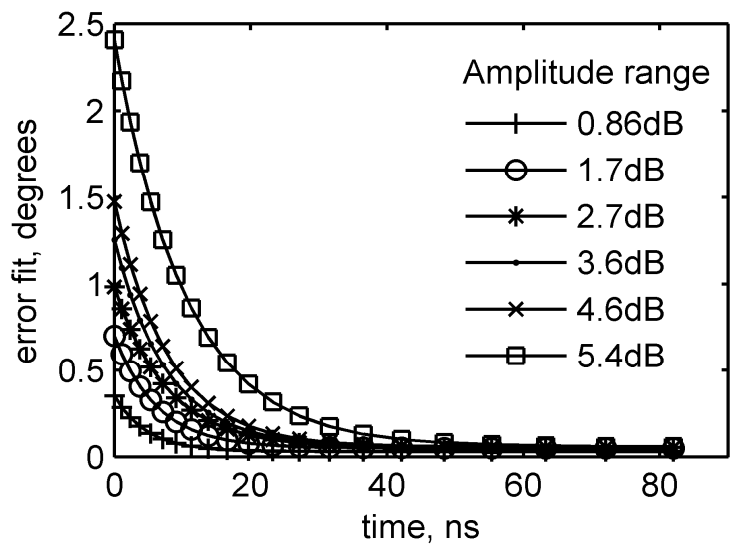

Fig. 4 Measured system alignment

\section{CONCLUSIONS}

We have demonstrated a resolution of better than $10 \mathrm{fs}$ for a beam timing measurement over a $9 \mathrm{~dB}$ amplitude dynamic range using a relatively straightforward heterodyne technique. The key component to obtaining the required noise performance was an array of eight analogue phase detectors.

An offline calibration procedure and post-detection software were used to correct for the amplitude dependent behaviour of the electronics. They permitted excellent alignment between two systems.

\section{ACKNOWLEDGEMENT}

We thank the CTF3 operations team for supplying the beams. This work is supported by the Commission of the European Communities under the 6th Framework Programme 'Structuring the European Research Area', contract number RIDS-011899.

\section{REFERENCES}

[1] GESCHONKE, G.: 'Status of the CLIC Test Facility (CTF3)', Proc. LINAC06, 21-25 August 2006, Knoxville, Tennessee, USA, pp. 11-15

[2] BRAUN, H.-H.: 'CLIC progress towards multi-TeV linear colliders', Proc. PAC05, 16-20 May 2005, Knoxville, Tennessee, USA, pp. 353-357

[3] ANDERSSON, A. and SLADEN, J. P. H.: 'First tests of a precision beam phase measurement system in CTF3', Proc. PAC07, 25-29 June 2007, Albuquerque, New Mexico, USA, pp. 302-304

[4] SYRATCHEV, I., SCHULTE, D., ADLI, E. and TABORELLI, M.: 'High RF power production for CLIC' Proc. PAC07, 25-29 June 2007, Albuquerque, New Mexico, USA, pp. 2194-2196 